Study of Condensed Matter by Neutron Scattering and Complementary Methods

\title{
Application of Modified Interference Wedge Method in Measurements of Indices of Refraction and Birefringence of Nematic Liquid Crystals
}

\author{
K. Kowiorski ${ }^{a}$, J. KęDzierski ${ }^{a}$, Z. RAszewski ${ }^{a}$, M.A. Kojdecki ${ }^{b, *}$, J. Herman ${ }^{c}$ \\ E. MISZCZYK ${ }^{d}$ AND W. PIECEK ${ }^{a}$ \\ ${ }^{a}$ Institute of Technical Physics, ${ }^{b}$ Institute of Mathematics and Cryptology, ${ }^{c}$ Institute of Chemistry \\ Military University of Technology, S. Kaliskiego 2, 00-908 Warszawa, Poland \\ ${ }^{d}$ Institute of Physics, University of Technology and Humanities, Malczewskiego 29, 26-100 Radom, Poland

\begin{abstract}
Wedge cell filled with a homogeneously oriented nematic liquid crystal was exploited as an interference wedge
\end{abstract} \\ to measuring indices of refraction. Interference fringes from the wedge were observed in polarised light in reflection \\ or transmission mode of a polarisation microscope to determining ordinary and extraordinary indices of refraction \\ or birefringence (i.e. anisotropy of both indices). The interference fringes close to wedge apex were uniform and \\ could be well described in two-beam interference approximation. The refractive indices were computed with using \\ simple formulae involving only distances between subsequent fringes in a cell filled with the liquid crystal and \\ an empty reference cell. Several new highly-birefringent nematic mixtures produced in the Military University of \\ Technology were investigated. The 6 CHBT nematic was used as the reference material.
}

DOI: $10.12693 /$ APhysPolA.124.946

PACS: 78.15.+e

\section{Introduction}

Light beam travelling through a uniaxial crystal is split into ordinary and extraordinary rays characterised by ordinary and extraordinary refractive indices. A plane wave of properly polarised light can propagate as only one of this rays. A homogeneously oriented nematic liquid crystal is an uniaxial crystal with optical axis of direction pointed by uniform nematic director. An ordinary ray of electric field vector perpendicular to the axis or an extraordinary ray of electric field vector parallel to the axis can be formed with using a polariser of eigenvector in corresponding direction. To determining refractive indices of liquid crystals, the same methods as for bulk crystals can be applied. The Haller method exploiting interference wedge, described in [1] and used in [2-5] was modified to measuring refractive indices of nematics.

A wedge cell (Fig. 1) were made of glass plates coated with transparent indium-tin oxide electrodes and polyimide films rubbed in direction parallel to wedge apex. The rectangular glass covers of size $22 \mathrm{~mm}$ per $35 \mathrm{~mm}$ were connected along short edge on one side and with the spacer $s$ of thickness about $0.12 \mathrm{~mm}$ on the other side. The cell was filled with a nematic liquid crystal. The rubbing enforced homogeneous orientation of the nematic with uniform director field in the direction parallel to both the wedge apex and plane surfaces of covers. Such cell was an optical wedge made of uniaxial crystal.

The interference fringes appear in polarised light transmitted through or reflected by the wedge cell. Each fringe

*corresponding author; e-mail: Marek.Kojdecki@wat.edu.pl

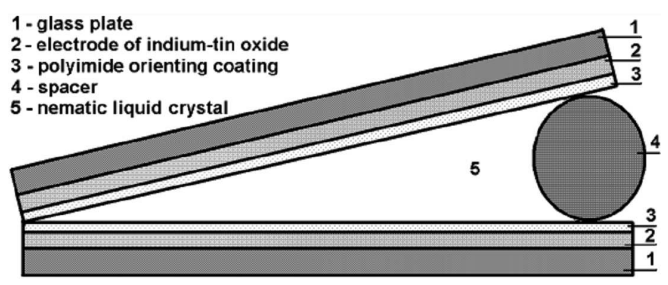

Fig. 1. Scheme of a wedge cell (side view).

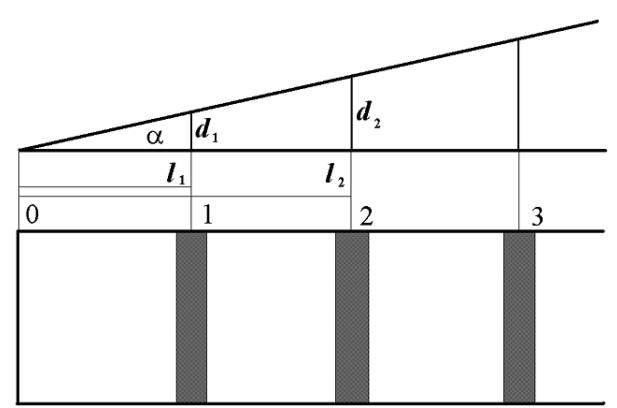

Fig. 2. Schematic sketch of interference fringes in a nematic wedge cell observed in polarised light (side view and front view).

corresponds to phase shift equal to an integer multiplicity of $\pi$ between the rays reflected from the surface of one and other cover; to describe well the fringes close to wedge apex it suffices to account for two-beam interference approximation. They can be observed in microscope.

In the reflection or transmission mode, light incident normally is polarised perpendicular or parallel to the wedge apex, which makes possible to determine ordinary or extraordinary index of refraction accordingly (Fig. 2). 


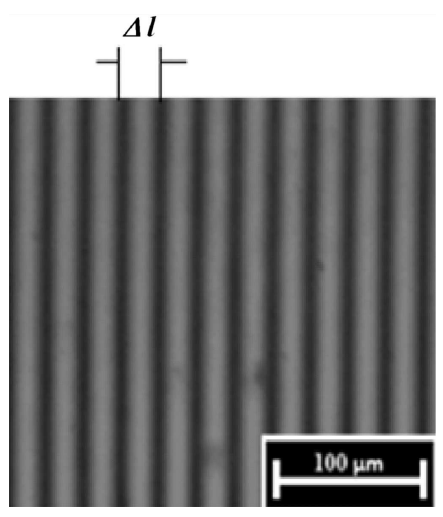

Fig. 3. Photograph of interference fringes in a wedge cell filled with 6CHBT observed in transmission mode in polarised light (sodium yellow line of wavelength of $589.3 \mathrm{~nm}$ ) at the temperature of $24^{\circ} \mathrm{C}$.

In the birefringence mode a light wave travels normally to the cell covers through the polariser before the cell and the analyser behind the cell, both ones of axes crossed at right angle and wedge apex being in diagonal position (Fig. 3). The relationship for distance $l_{k}$ from apex to a subsequent position of local maximum of intensity and corresponding cell thickness $d_{k}$ (in the fringe centre position) is the same as for distance $l$ to spacer of thickness $d$ :

$$
\frac{d}{l}=\frac{d_{1}}{l_{1}}=\ldots=\frac{d_{k}}{l_{k}}=\tan \alpha \approx \alpha
$$

with wedge apex angle $\alpha$, being of order of several milliradians (Fig. 2). In the reflection or transmission mode the $k$-th fringe in a cell with homogeneous filling with refractive index $n$, observed in normally incident polarised light of vacuum wavelength $\lambda$, appear in accordance with the condition for phase shift between rays reflected from one and other cell cover [1-8]:

$$
\frac{2 \pi}{\lambda} n d_{k}=\frac{2 \pi}{\lambda} \alpha n l_{k}=k \pi \text {. }
$$

Combining the formulae for two subsequent fringes for constant $\Delta l=l_{k+1}-l_{k}$, one obtains the basic relationship

$$
n=\frac{\lambda}{2 \alpha \Delta l}=\frac{\lambda l}{2 d \Delta l} .
$$

In the birefringence mode the last formula takes similar form $[5-8]$ :

$$
\Delta n=\frac{\lambda}{\alpha \Delta l},
$$

where $\Delta n=n_{\mathrm{e}}-n_{\mathrm{o}}$ is the difference of extraordinary and ordinary refractive indices (optical anisotropy or birefringence of a crystal filling the wedge).

\section{Method for measurement of refractive indices of liquid crystals}

The method is based on formulae (3) and (4) and relies on exploiting only constant distance between neighbour interference fringes observed in linearly polarised light. The minimal distance between fringes produced by an empty cell (filled with air), for which

$$
1=\frac{\lambda}{2 \alpha \Delta l_{a}},
$$

serves as the reference value. From formulae (3) and (5) a formula for corresponding refractive index follows: $n=$ $\Delta l_{a} / \Delta l$.

Measurements in interference system consisted of a wedge cell, polarisation microscope and micrometric eyepiece (OK15xKM) were realised in reflection mode, since the fringes were visible in reflected light much better than in transmitted light (it resulted from the dielectric coatings of the wedge cell featured small reflection coefficient) $[6,8,9]$. Firstly, the distance $\Delta l_{a}$ was measured for fringes in empty wedge cell (filled with air). Secondly, the cell was filled with a nematic liquid crystal oriented homogeneously parallel to the wedge apex and the measurements were done twice with using light polarised perpendicular or parallel to the wedge apex to determining $\Delta l_{\mathrm{o}}$ and $\Delta l_{\mathrm{e}}$ corresponding to ordinary and extraordinary refractive indices. Thirdly, the distance $\Delta l_{b}$ were determined in light transmitted through birefringent system consisting of microscope, polariser, cell, and analyser. Then the refractive indices $n_{\mathrm{o}}, n_{\mathrm{e}}, \Delta n$ were calculated independently from corresponding formulae

$$
n_{\mathrm{o}}=\frac{\Delta l_{a}}{\Delta l_{\mathrm{o}}}, \quad n_{\mathrm{e}}=\frac{\Delta l_{a}}{\Delta l_{\mathrm{e}}}, \quad \Delta n=\frac{2 \Delta l_{a}}{\Delta l_{b}} .
$$

\section{Experimental}

The method was used to determining refractive indices of seven liquid-crystalline mixtures (labelled further Mix1, Mix2, .., Mix8 and 1816) [10] and of the well-known nematic 6 CHBT as reference material. The chemical formulae are displayed in Fig. 4; the basic material was the mixture labelled 1816 . These mixtures are used in displays and integrated optics devices due to large optical anisotropy $(\Delta n)$. The results of measurements and computations are collected in Table. The results of measurements with using an Abbé refractometer for liquid crystals 1816 and 6 CHBT are added to compare both methods.
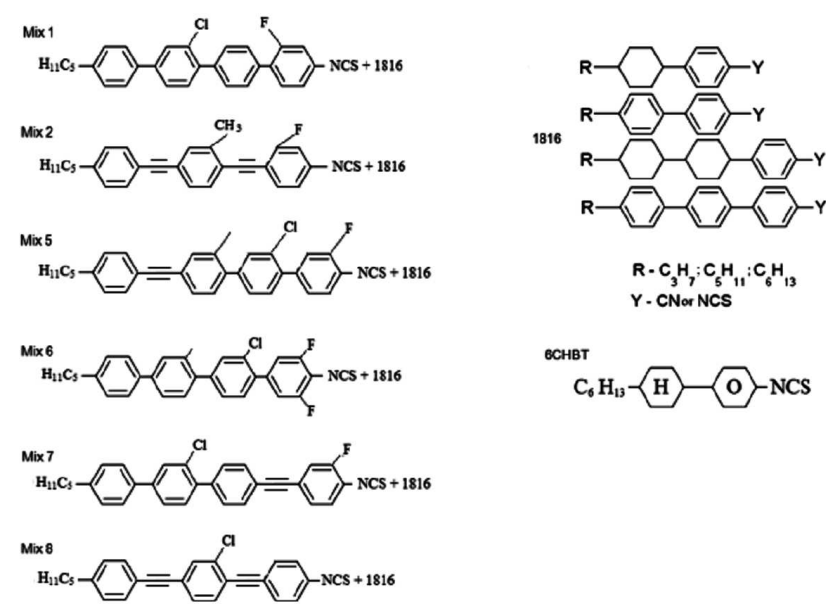

Fig. 4. Chemical formulae of nematic liquid crystals studied; basic material labelled 1816 is also a mixture. 
TABLE

Results of measurements for nematic liquid crystals under study obtained with using wedge cell method and with using an Abbé refractometer to compare.

\begin{tabular}{c|c|c|c|c|c|c}
\hline \hline \multirow{2}{*}{$\begin{array}{c}\text { Symbol of } \\
\text { substance }\end{array}$} & \multicolumn{2}{|c|}{ Wedge cell method } & \multicolumn{3}{c}{ Abbé refractometer } \\
\cline { 2 - 7 } & $\Delta n$ & $n_{\mathrm{e}}$ & $n_{\mathrm{o}}$ & $\Delta n$ & $n_{\mathrm{e}}$ & $n_{\mathrm{o}}$ \\
\hline 6CHBT & 0.157 & 1.675 & 1.518 & 0.157 & 1.677 & 1.520 \\
1816 & 0.220 & 1.721 & 1.501 & 0.221 & 1.724 & 1.503 \\
Mix1 & 0.235 & 1.728 & 1.493 & & & \\
Mix2 & 0.251 & 1.784 & 1.533 & & & \\
Mix5 & 0.238 & 1.758 & 1.520 & & & \\
Mix6 & 0.234 & 1.693 & 1.459 & & & \\
Mix7 & 0.242 & 1.762 & 1.520 & & & \\
Mix8 & 0.246 & 1.723 & 1.477 & & &
\end{tabular}

\section{Discussion}

The described above method for determining refractive indices of liquid crystals can be effectively used only when the interference fringes are equidistant. This is the case when the reflection coefficient at the limit of cell cover and liquid crystal is small [5] and the two-beam approximation for describing interference is accurate. Then in general at least a few tens of fringes close to wedge cell apex are equidistant in good approximation and only up to about twenty should be exploited in measurements. In the birefringent system all fringes observed in transmitted light are then equidistant [7]. For successful measurements the following conditions should be preserved:

- The spacer should be chosen of size enabling rather narrow fringes, since for broad fringes the distance between them is determined less precisely.

- The interference wedge cell should be constructed of glass plates with refractive index sufficient to fulfilling Fresnel conditions for reflection from limits of liquid crystal $[6,8]$.

- The cell for measurements can be also cylindrical or spherical [11-13]; then formulae (6) must be modified.

- Highly monochromatic light is strongly recommended for stable measurements. In this work yellow light from sodium lamp was applied. Coherent laser light is worse due to coherence noise disturbing measurements.

- The determination of birefringence $(\Delta n)$ in dependence on temperature is enough to estimating the parameter of orientation order of liquid crystal [11-13].

More accurate multi-beam approximation of interference together with methods for determining refractive indices, exploiting distances between interference fringes, was described in [5]. The determination of birefringence is less precise than in the case of present method.

\section{Conclusion}

The simple method was described for determining ordinary and extraordinary refractive indices and birefringence of uniaxial liquid crystals from only distances between interference fringes observed in an empty and then filled wedge cell. The method was verified successfully in measurements of parameters of new liquid crystalline mixtures.

\section{Acknowledgments}

This paper was supported by NCBIR, Poland under grant PBS1/B3/9/2012 for " $5 \mathrm{D}$ interferometer for the machinery geometry monitoring".

\section{References}

[1] I. Haller, H.A. Huggins, M.J. Freiser, Mol. Cryst. Liq. Cryst. 16, 53 (1972).

[2] J.W. Baran, J. Kędzierski, Z. Raszewski, J. Żmija, Biuletyn WAT 25, 147 (1976) (in Polish).

[3] J.W. Baran, J. Kędzierski, Z. Raszewski, J. Żmija, Materiaty Elektroniczne 2, 7 (1978) (in Polish).

[4] J.W. Baran, F. Borowski, J. Kędzierski, Z. Raszewski, J. Żmija, K. Sadowska, Bull. Acad. Sci. Pol. 26, 117 (1978).

[5] J. Kędzierski, Z. Raszewski, M.A. Kojdecki, E. Kruszelnicki-Nowinowski, P. Perkowski, W. Piecek, E. Miszczyk, J. Zieliński, P. Morawiak, K. Ogrodnik, Opto-Electron. Rev. 18, 214 (2010).

[6] M. Pluta, Optical Microscopy, PWN, Warszawa 1982 (in Polish).

[7] M. Pluta, Microinterferometry in Polarized Light, WNT, Warszawa 1991 (in Polish).

[8] M. Pluta, Advanced Light Microscopy, Vol. I (Principles and Basic Properties), Vol. II (Specialized Methods), Vol. III (Measuring Techniques), PWN, Warszawa 1988-1989-1993.

[9] S. Tolanski, Multiple-Beam Interference Microscopy of Metals, Academic Press, London 1970.

[10] J. Herman, J. Dziaduszek, R. Dąbrowski, J. Kędzierski, K. Kowiorski, V. Sai Dasari, S. Dhara, P. Kula, Liq. Cryst. 40, 1174 (2013).

[11] B. Żywucki, W. Kuczyński, G. Czechowski, Proc. SPIE 2372, 151 (1995).

[12] B. Żywucki, W. Kuczyński, IEEE Trans. Diel. Electr. Insul. 8, 512 (2001).

[13] W. Kuczyński, B. Żywucki, J. Małecki, Mol. Cryst. Liq. Cryst. 381, 1 (2002). 